\title{
Development of practical functional electrical stimulation cycling systems based on an electromyography study of the Cybathlon 2016
}

\author{
Jetsada Arnin, Traisak Yamsa-ard, Preechapawan Triponyuwasin, Yodchanan Wongsawat
}

Department of Biomedical Engineering, Faculty of Engineering, Mahidol University

This article is distributed under the terms of the Creative Commons Attribution Noncommercial License (CC BY-NC 4.0) which permits any noncommercial use, distribution, and reproduction in any medium, provided the original author(s) and source are credited.

\begin{abstract}
The purpose of this study was to develop a functional electrical stimulation (FES) system based on the motor driving concept for use by spinal cord injury patients participating in the FES Cycling competition at the Cybathlon 2016. The proposed FES system consists of a low-power control system, a precise processor unit, and a 4-channel stimulation unit. Self-adhesive carbon conductive electrodes were utilized for stimulation. A 26-year-old SCI patient was qualified to participate in the competition. The pilot patient underwent training for 16 months, which included experience with FES stimulation, performing FES cycling, and reducing spasticity, to practice using the FES system. In addition, using surface electromyography (EMG) during cycling, the muscle activation pattern for generating the stimulation profile was applied and resulted in good performance. The best FES cycling performance the pilot achieved was 1000 meters translation with the cycling system during twelve minutes of using the FES system. The pilot achieved an 1000 meters translation mobility within an average of 16 minutes of cycling. Nevertheless, the system must be further investigated regarding muscle fatigue and other factors that may affect the stimulation conditions.
\end{abstract}

Key Words: Functional Electrical Stimulation, Spinal Cord Injury, Voltage Stimulation, EMG, FES Cycling

Eur J Transl Myol 27 (4): 295-301

The number of spinal cord injury (SCI) patients is growing each year. The main causes of the injury are vehicle crashes, followed by falls and acts of violence. ${ }^{1}$ SCI results in various adverse effects, ${ }^{2}$ including loss of walking ability, impaired postural control, loss of muscle strength, limit activity daily livings (ADLs) function, all of which initiate disability and long-term complications. ${ }^{3}$ Although many SCI research groups are continuously working to develop novel treatments, such as prostheses and alternative medications, that may support nerve cell rejuvenation, ${ }^{4}$ or improve the function of the nerves that persist after a SCI, ${ }^{5}$ the recovery outcomes were still not satisfied. Thus, current interventions for SCI primarily focus on preventing further injury and empowering people with SCI to recover. ${ }^{6}$ Designing assistive technologies that meet the requirements of being suitably sufficient, cosmetically pleasing, and cost effective remains a challenge. As there is a need for improved rehabilitation technology, more research and development in this field is required to improve the quality of life of these patients. ${ }^{7}$ For example, allowing patients to engage in activities depends on matching and fitting the wheelchair design. Regarding assistive technologies, FES is a powerful tool for augmenting the rehabilitation outcomes of SCI patients. ${ }^{8,9}$ FES technology significantly advanced thus far in the development of rehabilitation technologies and improving our understanding of the muscle-nerve relationship. ${ }^{10}$ However, applications of implementing FES devices into individual rehabilitation therapies for these patients still has some limitations. ${ }^{11}$ FES technologies have major applications in various therapies, including drop foot stimulation, ${ }^{12}$ gait training combined with FES, ${ }^{13}$ and even upper-limb or lowerlimb rehabilitation. ${ }^{14,15}$ In some paraplegic patients, FES have been extensively considered as a tool to restore walking function, although implementing such devices is practically difficult because there are numerous degrees of freedom related to walking. ${ }^{16}$ Hence FES cycling was developed for these patients, and its advantages can be preserved for extended periods of time using trained muscles. ${ }^{17}$ In addition, previous studies have shown significant relationships between EMG signals and FES during cycling. ${ }^{18-20}$ These studies revealed the contribution of stimulation voltage and patterns, which can be supplementary applied for practical use, although the conditions of muscle fatigue need to be further investigated. Regarding FES 


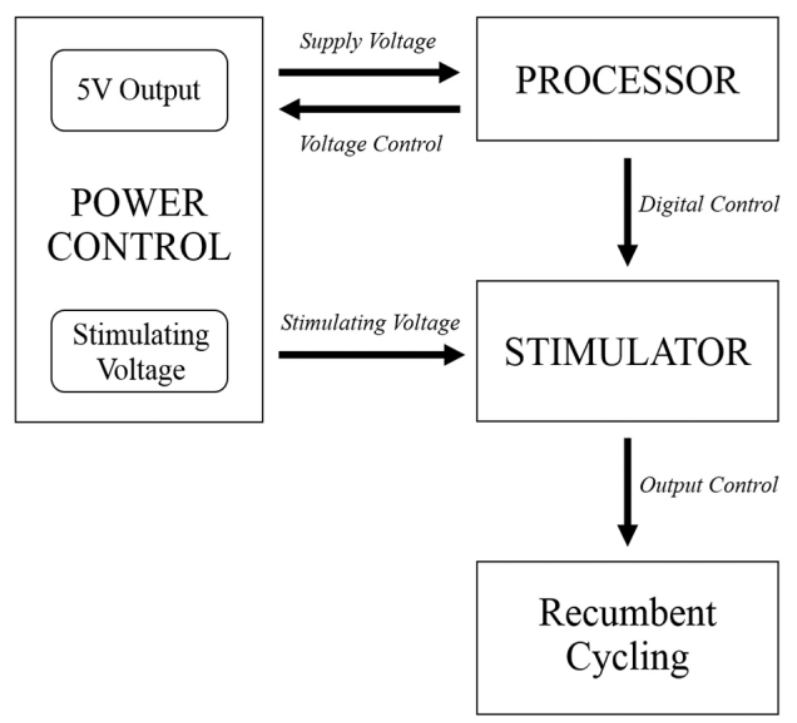

Fig 1. An Overview of the Proposed FES System

cycling, ${ }^{21-23}$ many studies have shown the importance of the effective application of FES cycling including the effects of muscle size, strength and function as well as cardiovascular and bone changes. In practice, FES cycling and other FES exercises such as FES rowing involve the use of surface electrodes, intensive training, determining stimulation limitations, and implanted stimulators. In this paper, a FES cycling stimulator and a stimulation technique are proposed for practical use by a patient competing in the Cybathlon 2016. EMG patterns were studied for implementation to generate individual stimulation patterns. As an inexpensive system design, the device can be feasibly mass produced. In addition, pilot information, bike information, electrical stimulation, training technique, and performance were evaluated. Some information here presented was submitted to IFESS Conference 2016 and have been accepted for oral presentation duringin the Special Session "FES Cycling".

\section{Pilot information and FES System}

\section{Design of the Proposed FES Device}

In this work, we designed a functional electrical stimulation device that can be efficiently used for controlling a recumbent bike. The FES device was

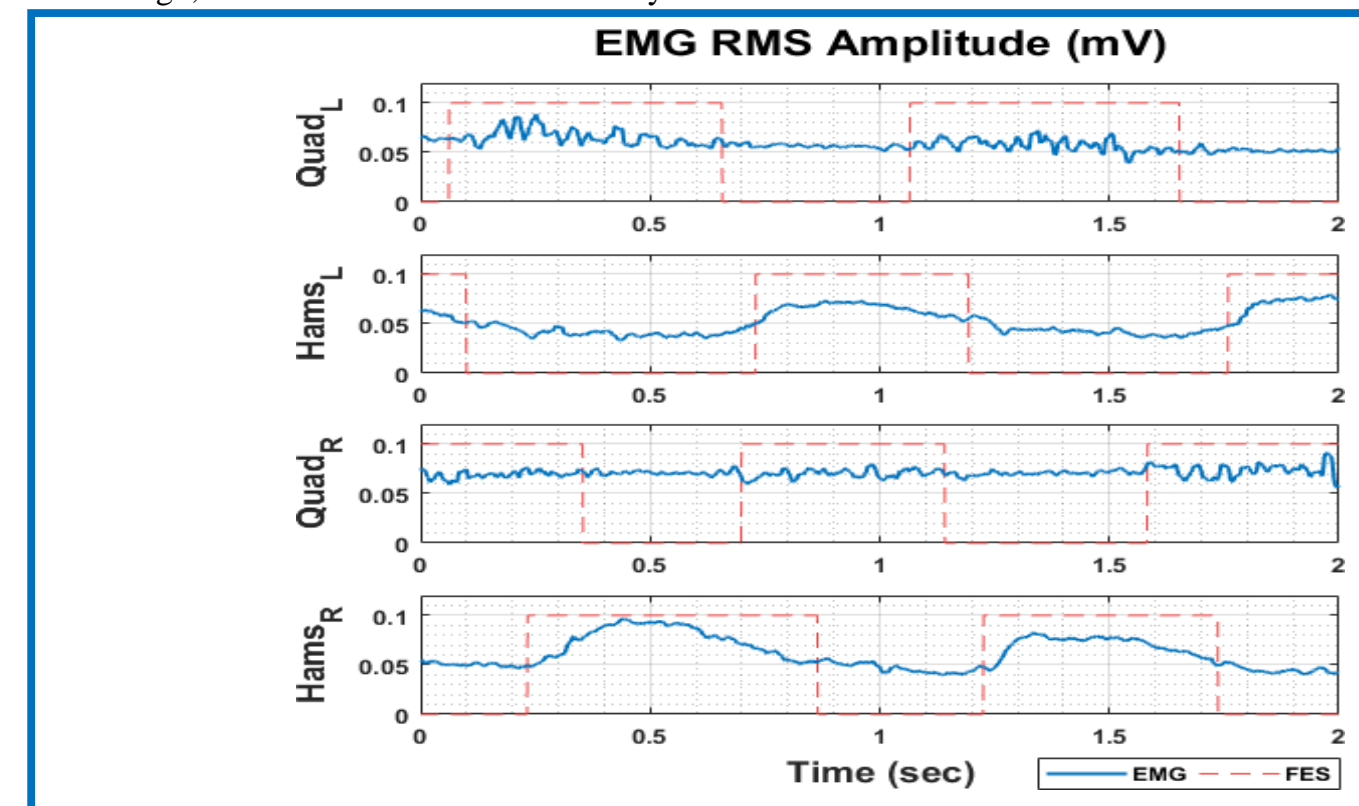

Fig 2. EMG during Cycling and Default FES Sequence of Subject 2 
designed to deliver non-uniform stimulation according to the patterns of muscle contraction during cycling. Over the past decade, many studies of EMG patterns have been reported. ${ }^{24}$ According to previous studies, ${ }^{25-6}$ the normalized cycling patterns were related to the EMG power recorded from different muscles. This EMG study was designed to explore cycling patterns during use of a recumbent bike, which were then used as a default activation sequence. Five healthy participants were asked to perform bicycling, and their EMGs were recorded (more details below). The study focused on finding the relationship between EMG power and cycling phrases. Another important feature of our system is the circuit design. In general, optimal FES circuits should have small components, and low input voltage is required. ${ }^{27}$ In this project, we assembled a customized circuit based on a previous design. ${ }^{28,29}$ An overview of the proposed system is shown in Figure 1. The FES device consists of three parts, as follows:

1) The voltage generator: This component generates voltages each channel from $5 \mathrm{~V}$ to $45 \mathrm{~V}$. The power management component was composed of 3 parts. The first part is a step-down voltage regulator used to maintain a $5 \mathrm{~V}$ supply, which delivers power to the peripheral component. The second part is a 5-45 $\mathrm{V}$ adjustable step-up regulator used to supply the stimulation module. During cycling, we analyzed the duration of the stimulation pulse from the absolute power of the EMG in each phase of cycling. In the initial phase, the pulse duration would last longer than in other phases in order to accumulate muscle forces. In the Cybathlon competition, we deployed a dynamic duration ranging from 200 to $600 \mu \mathrm{s}$. The output voltage can be adjusted by a knob located at the hand grips on both sides.

2) The processor unit: This unit generates the pulse sequences for stimulation. The processor manipulates the stimulation waveform by configuring parameters, including frequency, duty cycle and output voltage. This dynamic waveform is based on a biphasic square wave. The frequency of

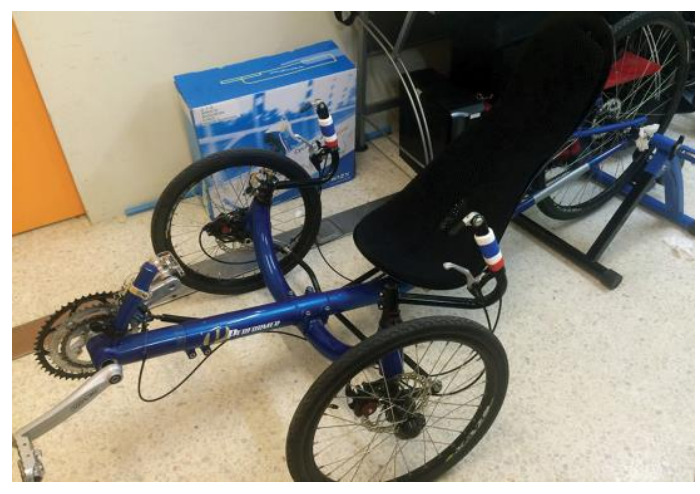

Fig 3. FES-integrated Recumbent Bike the waveform is related to the cycling period, since a muscle contracts at different forces in different phases of cycling. The bandwidth of the stimulation frequency is $20-50 \mathrm{~Hz}$.

3) The stimulation module: The last module, a voltage control stimulator, incorporates 4-channel stimulation with user-configurable settings that include frequency, duty cycle, and voltage. For safety considerations, each channel generates a maximum current of $80 \mathrm{~mA}$ for a $560 \Omega$ load combined with voltage surge and current leakage protection. In addition, to determine the stimulation pattern, the root-mean-square (RMS) of the EMG signal was utilized to justify the cycling patterns. The RMS values of EMGs recorded from the quadriceps and hamstring of both the left and right legs were computed, and then a thresholding method was used to generate the stimulation pattern. The example of the default activation sequence is shown in Figure 2. The parameters of each channel, including pulse width and amplitude, can be separately tuned for different muscle targets and cycling phases.

Regarding the stimulation system, 2 in $\times 4$ in selfadhesive carbon conductive pads were used as electrodes due to the large muscle areas. The electrodes were placed on the rectus femoris and hamstring on both sides. The stimulation system is an open-loop userselective system that includes both automatic control and manual control. For automatic control, the stimulation patterns referred to in the previous study are automatically generated using subject-specific cycling phrases, which require individual calibration and fine tuning before use. Inversely, for manual control, the user must activate a trigger located on the hand grips to generate the stimulation pulse independently on each side. Therefore, the user must first undergo a training session to familiarize him/herself with the system.

\section{Selected Recumbent Bike}

The major concerns regarding selection of the bike are

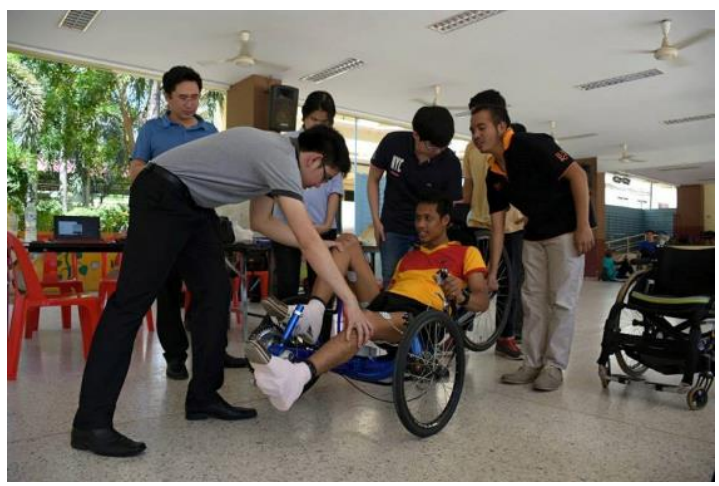

Fig 4. The First FES Cycling Test 
weight and embarkation. In this regard, we selected a bike frame made of aluminum alloys, with an average weight of $18.4 \mathrm{~kg}$ and a $120-\mathrm{kg}$ rider weight limit. The bike included 27 adjustable speeds with 9 back cogs and 3 front chain rings. The wheels were Kenda K-193 wheels sized 20" x $1.25700 \mathrm{c}$ x 32c. The pilot seat was constructed of fiber-reinforced plastics (FRP). Figure 3 illustrates the recumbent with the training equipment. During training and competition, we used the lowest force of gear driving. Moreover, after the final rehearsal of the Cybathlon in June 2015 at Zurich, Switzerland, we re-designed the pedals to hold pilot's legs for safety. An aluminum composite plate, which can be tightened by a strip, was used to modify the bike. It should be noted that there was no instrumentation device attached to the bike.

\section{Pilot Details}

In Thailand, there are many nonprofit organizations dedicated to caring for people with disabilities. Disabled patients from these organizations were willing to be subjects in this research. Three criteria were used to select a participant: intention of the subject to practice and be disciplined, a level of injury that completely meets the criteria for participation in the Cybathlon competition, and muscle spasticity. The muscle must be contracted enough to lift their limbs when stimulated by the FES device. The selected pilot was a 26-year-old man with T4-level lesion. His weight and height are 165 $\mathrm{cm}$ and $62 \mathrm{~kg}$, respectively. He was recruited 3 years ago at the Redemptorist Foundation for People with Disabilities (RFPD), Thailand. He had been using a wheelchair for more than 5 years and was in a healthy condition. The pilot entirely passed the special medical examination according to the Cybathlon criteria. It should be noted that during the competition, the pilot is allowed to use only the FES without any devices to monitor vital signs.

\section{FES Training Protocol}

The pilot practiced using the FES system in four training phases. The first phases focused on familiarity of using the FES system. Initially, the pilot was assigned to daily stimulation of the main muscles, i.e., the rectus femoris and hamstrings, at the lowest voltage level that was able to lift the limbs up and hold them raised for 5 seconds; the muscles then rested for 10 seconds, and the procedure was repeated. This session lasted until fatigue occurred, which was evaluated by a physician. The training was immediately stopped if fatigue occurred. After finishing the session, the physician helped the patient to perform stretching exercises to reduce spasticity. Training was begun in 2015 January and lasted 6 months. The primary limitation of training was time constraints, as the pilot could only train once per week due to his family obligations and transportation. The second training phase aimed to allow the pilot to perform cycling using the FES device. The pilot was trained to cycle in a recumbent position. For the pilot's safety, a cycling stand was used for practicing, as shown in Figure 4. In this stage, we found that the pilot had a clonus condition which occasionally obstructed the training. As his ankle was sometimes in a dorsiflexion position, he sometimes exhibited involuntary muscle contraction. Whenever a clonus condition occurred, the stimulation was terminated because it affected the cycling rhythm. His muscles could not be stimulated until his clonus was recovered. The third training phase comprised analyzing the EMG power to optimize the stimulus voltage for muscle contraction during cycling. Two experiments were conducted in five healthy participants. First, the EMG activity of muscle contractions of the rectus femoris and hamstrings was recorded during cycling. EMG activity was recorded using a BIOPAC MP36 system (BIOPAC Systems, USA) with a gain of 1000 and a sampling frequency of $1 \mathrm{kHz}$. Analog signal preprocessing of the EMG signals included a $10-500 \mathrm{~Hz}$ bandpass filter and a $50 \mathrm{~Hz}$ notch filter. The rules of the Cybathlon competition state that the pilot must perform cycling for 750 meters within 8 minutes. Therefore, participants were assigned to cycle for 8 minutes and maintain a cycling speed of $60 \mathrm{rpm}$ according to the minimum speed of the bike at the lowest driving gear. EMG recording was begun after the participant reached a speed of $60 \mathrm{rpm}$. The results were converted to EMG RMS amplitude, and then a thresholding method was used to obtain the FES patterns for a default activation sequence, as shown in Figure 2. A second experiment was conducted to find the optimal stimulation voltage level that is suited for the best cycling performance. Both stimulation electrodes and recording electrodes were placed over the hamstring and quadriceps muscles on the participants. The stimulation voltage was manually adjusted starting from $10 \mathrm{~V}$ and increased to $30 \mathrm{~V}$ in steps of $1 \mathrm{~V}$. If the participants felt uncomfortable at any point during the voltage stimulation, the stimulation was terminated. However, the high-voltage stimulation pulses still interfere with the EMG recording, resulting in signal saturation. To reduce the interference, we used an adaptive algorithm based on a Gram-Schmidt prediction error filter (GSPEF) ${ }^{30}$ The EMG RMS was subsequently calculated as a function of the stimulation voltage. The voltage that yielded $80 \%$ of the maximum RMS was used as the default stimulation voltage. As a result, the stimulation voltage is relative to the individual; for example, the pilot required stimulation voltages of $28 \mathrm{~V}$ and $33 \mathrm{~V}$ for the left and right quadriceps and $30 \mathrm{~V}$ and $33 \mathrm{~V}$ for the left and right hamstring, respectively. Other stimulation characteristics were defined according to previous studies that evaluated EMG patterns during cycling. ${ }^{25,26}$ The final training phase was incorporated to strengthen the muscles and reduce spasticity and to find the optimal approach to minimize the possibility of clonus. The pilot patient was assigned to daily physical exercise 


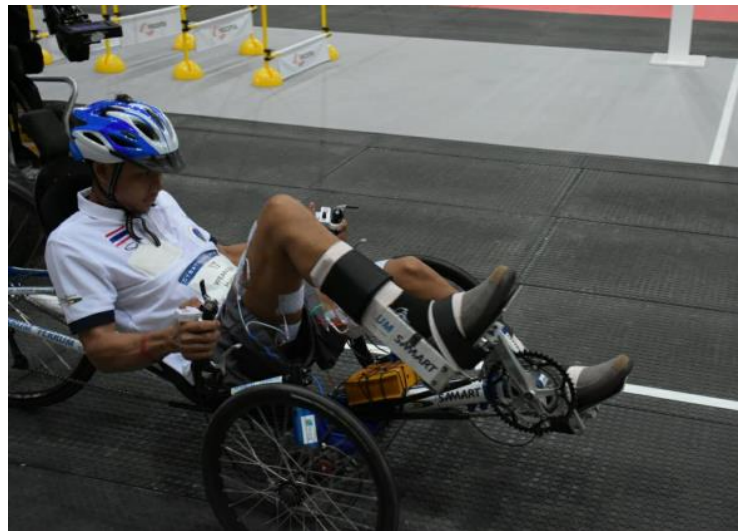

Fig 5. Pilot Performing in the Cybathlon

using FES to stimulate his muscles to lift up a sand bag, which was stopped when he felt his muscle was going to fatigue.

\section{Performance}

We propose flexible stimulation patterns for which parameters can be tuned and individualized for specific users. However, there are marked differences between healthy subjects and the paraplegia cases that need to be further investigated, e.g., proper stimulation voltage and pulse width.17 Furthermore, fatigue is a major concern for long-term use of FES. ${ }^{31-32}$ After more than 16 months of practice in recumbent cycling using the 4channel FES device, the best performance achieved by the pilot was cycling 1000 meters in translation in twelve minutes. The average cycling speed was 1000 meters of translation in 16 minutes. Unfortunately, the pilot exhibited unpredictable clonus in the Cybathlon. Although we dedicated marked efforts to addressing the issue, it was ultimately not well managed.

\section{Concluding Remarks}

The use of EMG patterns in FES cycling stimulation was proposed herein. This approach was implemented in an SCI patient and yielded a good result. It should be noted that the fatiguing condition needs to be further investigated to obtain the appropriate stimulation voltage and pattern. During the practice session, the pilot exhibited satisfactory performance, although he was not successful in the competition. Nonetheless, the developed 4-channel FES device was compact, efficient, and stable. Collaboration with other teams present at the first Cybathlon FES Cycling competition and beyond will provide further hints and tips. ${ }^{33-40}$ In the future, we plan to implement more stimulation channels that would increase flexibility and improve performance for individual usage while incorporating a user-friendly, aesthetic, easy to handle design.

\section{List of acronyms}

ADLs - Activity Daily Livings

EMG - Electromyography
FES - Functional Electrical Stimulation

FRP - fiber-reinforced plastics

RMS - root-mean-square

RFPD - Redemptorist Foundation for People with

Disabilities

SCI - Spinal Cord Injury

\section{Author's contributions}

All authors contributed equally to this work. Jetsada Arnin, Traisak Yamsa-ard, and Preechapawan Triponyuwasin designed the study, performed the experiments, analyzed the data, finalized the working prototype, and prepared the manuscript. Yodchanan Wongsawat discussed the results and implications and commented on all stages of the manuscript.

\section{Acknowledgments}

This work was supported in part by the National Research Council of Thailand (NRCT). The authors would like to acknowledge all the researchers of the Brain-Computer Interface Laboratory (BCI LAB), Faculty of Engineering, Mahidol University for their support and the Golden Jubilee Medical Center for coordinating with the spinal cord injury patient.Dr. Wongsawat reports grants from The National Research Council of Thailand (NRCT) during the study; other from the Cybathlon Rehearsal 2015, outside the submitted work.

\section{Conflict of Interest}

There are no competing interests to report.

\section{Ethical Publication Statement}

All procedures were approved the IRB at the BrainComputer Interface Laboratory, Faculty of Engineering, Mahidol University (ethics certificate of approval number: MU-CIRB 2016/042.2803). All subjects signed consent forms and provided consent to publish their pictures. We have read the Journal's position on issues involved in ethical publication and we confirm that this report is consistent with those guidelines.

\section{Corresponding Author}

Yodchanan Wongsawat, Department of Biomedical Engineering, Faculty of Engineering, Mahidol University 25/25 Putttamonthon 4 Rd., Salaya, Nakorn Pathom 73170, Thailand phone: 662-441-4255; fax: 662-441-4254.

E-mail: yodchanan.won@mahidol.ac.th

\section{E-mails of co-authors}

Jetsada Arnin: jetsada.arn@mahidol.ac.th Traisak Yamsa-ard: traisak.yam@mahidol.edu Preechapawan Triponyuwasin: preechapawan.tri@mahidol.edu

\section{References}

1. Spinal cord injury facts and figures at a Glance. J Spinal Cord Med 2012;35:197-8. 
2. Takeuchi N, Izumi SI. Rehabilitation with poststroke motor recovery: a review with a focus on neural plasticity. Stroke Res Treat 2013.

3. McKinley WO, Jackson AB, Cardenas DD, Michael J. Long-term medical complications after traumatic spinal cord injury: a regional model systems analysis. Arch Phys Med Rehabil. 1999;80:1402-10.

4. Schwab ME. Repairing the injured spinal cord. Science. 2002;295:1029-31.

5. Ramón-Cueto A, Cordero MI, Santos-Benito FF, Avila J. Functional recovery of paraplegic rats and motor axon regeneration in their spinal cords by olfactory ensheathing glia. Neuron 2000;25:42535.

6. Maynard FM, Bracken MB, Creasey GJ, et al. International standards for neurological and functional classification of spinal cord injury. Spinal cord 1997;35:266-74.

7. Sisto SA, Forrest GF, Faghri PD. Technology for mobility and quality of life in spinal cord injury [Analyzing a Series of Options Available]. IEEE Eng Med Biol Mag 2008;27(2).

8. Debelle A, Hermans L, Bosquet M, et al. Soft Encapsulation of Flexible Electrical Stimulation Implant: Challenges and Innovations. Eur J Transl Myol 2016;26(4):6298.

9. Popović DB. Advances in functional electrical stimulation (FES). J Electromyogr Kinesiol 2014;24:795-802.

10. Coste CA, Mayr W, Bijak M, et al. FES in Europe and Beyond: Current Translational Research. Eur J Transl Myol 2016;26(4):6369.

11. Valtin M, Kociemba K, Behling C, et al. RehaMovePro: A Versatile Mobile Stimulation System for Transcutaneous FES Applications. Eur J Transl Myol 2016;26(3):6076.

12. Lyons GM, Sinkjær T, Burridge JH, Wilcox DJ. A review of portable FES-based neural orthoses for the correction of drop foot. IEEE Trans Neural Syst Rehabil Eng 2002;10:260-79.

13. Cho MK, Kim JH, Chung Y, Hwang S. Treadmill gait training combined with functional electrical stimulation on hip abductor and ankle dorsiflexor muscles for chronic hemiparesis. Gait posture 2015;42:73-8.

14. Kim H, Lee G, Song C. Effect of functional electrical stimulation with mirror therapy on upper extremity motor function in poststroke patients. $\mathrm{J}$ Stroke Cerebrovasc Dis 2014;23:655-61.

15. Kutlu M, Freeman CT, Hallewell E, et al. Upperlimb stroke rehabilitation using electrode-array based functional electrical stimulation with sensing and control innovations. Med Eng Phys 2016;38:366-79.

16. Abdulla SC, Sayidmarie O, Tokhi MO. Functional electrical stimulation-based cycling assisted by flywheel and electrical clutch mechanism: A feasibility simulation study. Rob Auton Syst 2014;62:188-99.

17. Guimarães JA, da Fonseca LO, Dos Santos-CoutoPaz CC, et al. Towards Parameters and Protocols to Recommend FES-Cycling in Cases of Paraplegia: A Preliminary Report. Eur J Transl Myol 2016;26(3):6085.

18. Lauer RT, Johnston TE, Smith BT, Lee SC. Lower extremity muscle activity during cycling in adolescents with and without cerebral palsy. Clin Biomech 2008;23:442-9.

19. Roth N, Wiener A, Mizrahi J. Methods for Dynamic Characterization of the Major Muscles Activating the Lower Limb Joints in Cycling Motion. Eur J Transl Myol 2014;24(3):3317.

20. Hayashibe M, Zhang Q, Guiraud D, Fattal C. Evoked EMG-based torque prediction under muscle fatigue in implanted neural stimulation. J Neural Eng 2011;8:064001.

21. Newham DJ, Donaldson ND. FES cycling. Operative Neuromodulation 2007:395-402.

22. Frotzler A, Coupaud S, Perret C, et al. Highvolume FES-cycling partially reverses bone loss in people with chronic spinal cord injury. Bone. 2008;43:169-76.

23. Chen K, Chen SC, Tsai KH, et al. An improved design of home cycling system via functional electrical stimulation for paraplegics. Int $\mathrm{J}$ Ind Ergon. 2004;34:223-35.

24. Hug F, Dorel S. Electromyographic analysis of pedaling: a review. J Electromyogr Kinesiol. 2009;19:182-98.

25. Rouffet DM, Hautier CA. EMG normalization to study muscle activation in cycling. J Electromyogr Kinesiol. 2008;18:866-78.

26. Prilutsky BI, Gregor RJ. Analysis of muscle coordination strategies in cycling. IEEE Trans Rehabil Eng. 2000;8:362-70.

27. Stein RB, inventor; Neuromotion Inc., assignee. Assembly for functional electrical stimulation during movement. United States patent US 5,814,093. 1998.

28. Cheng KE, Lu Y, Tong KY, et al. Development of a circuit for functional electrical stimulation. IEEE Trans Neural Syst Rehabil Eng. 2004;12:43-7.

29. Doucet BM, Lam A, Griffin L. Neuromuscular electrical stimulation for skeletal muscle function. Yale J Biol Med. 2012;85:201.

30. Yeom H, Chang YH. Autogenic EMG-controlled functional electrical stimulation for ankle dorsiflexion control. J Neurosci Methods. 2010;193:118-25.

31. Karu ZZ, Durfee WK, Barzilai AM. Reducing muscle fatigue in FES applications by stimulating with N-let pulse trains. IEEE Trans Biomed Eng. 1995;42:809-17.

32. Eser PC, Donaldson NN, Knecht H, Stussi E. Influence of different stimulation frequencies on 
power output and fatigue during FES-cycling in recently injured SCI people. IEEE Trans Neural Syst Rehabil Eng. 2003;11:236-40.

33. Azevedo Coste C, Bergeron V, Berkelman R, et al. Comparison of strategies and performance of functional electrical stimulation cycling in spinal cord injury pilots for competition in the first ever CYBATHLON. Eur J Transl Myol 2017;27:251-4.

34. Berkelmans R, Woods B. Strategies and performances of Functional Electrical Stimulation Cycling using the BerkelBike with Spinal Cord Injury in a competition context (CYBATHLON). Eur J Transl Myol 2017;27:255-8.

35. Laubacher M, Aksöz EA, Bersch I, Hunt KJ. The road to Cybathlon 2016 - Functional electrical stimulation cycling Team IRPT/SPZ. Eur J Transl Myol 2017;27:259-64.

36. Sijobert B, Fattal C, Daubigney A, AzevedoCoste B. Participation to the first Cybathlon: an overview of the FREEWHEELS team FES-cycling solution. Eur J Transl Myol 2017;27:265-71.
37. Guimarães JA, Oliveira da Fonseca L, de Sousa AC, et al. FES Bike Race preparation to Cybathlon 2016 by EMA team: a short case report. Eur J Transl Myol 2017;27:272-78.

38. Metani A, Popović-Maneski L, Mateo S, et al. Functional electrical stimulation cycling strategies tested during preparation for the First Cybathlon Competition - a practical report from team ENS de Lyon. Eur J Transl Myol 2017;27:279-88.

39. McDaniel J, Lombardo LM, Foglyano KM, et al. Cycle Training Using Implanted Neural Prostheses: Team Cleveland. Eur J Transl Myol 2017;27:289-94.

40. Leung WC, Tong RKY, Wang X, et al. The Effectiveness of Functional Electrical Stimulation (FES) in On-Off Mode for enhancing the cycling performance of Team Phoenix at 2016 Cybathlon. Eur J Transl Myo 2017;27:302-6. 